\title{
Hemodynamic goal-directed therapy and postoperative kidney injury: an updated meta-analysis with trial sequential analysis
}

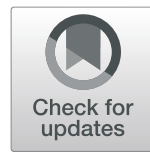

\author{
Mariateresa Giglio* ${ }^{*}$, Lidia Dalfino, Filomena Puntillo and Nicola Brienza
}

\begin{abstract}
Background: Perioperative goal-directed therapy (GDT) reduces the risk of renal injury. However, several questions remain unanswered, such as target, kind of patients and surgery, and role of fluids and inotropes. We therefore update a previous analysis, including all studies published in the meanwhile, to clarify the clinical impact of this strategy on acute kidney injury.

Main body: Randomized controlled trials enrolling adult patients undergoing major surgery were considered. GDT was defined as perioperative monitoring and manipulation of hemodynamic parameters to reach normal or supranormal values by fluids alone or with inotropes. Trials comparing the effects of GDT and standard hemodynamic therapy were considered. Primary outcome was acute kidney injury, whichever definition was used. Meta-analytic techniques (analysis software RevMan, version 5.3) were used to combine studies, using random-effect odds ratios (OR) and 95\% confidence intervals (Cl). Trial sequential analyses were performed including all trials and considering only low risk of bias trials. Sixty-five trials with an overall sample of 9308 patients were included. OR for the development of renal injury was $0.64(95 \% \mathrm{Cl}, 0.62-0.87 ; p=0.0003)$, with no statistical heterogeneity. Trial sequential analyses and sensitivity analysis including studies with low risk of bias confirmed the main results. A significant decrease in renal injury rate was observed in studies that adopted cardiac output and oxygen delivery as hemodynamic target and that used both fluids and inotropes. The postoperative kidney injury rate was significantly lower in trials enrolling "high-risk" patients and major abdominal and orthopedic surgery.

Short conclusion: The present meta-analysis suggests that targeting GDT to perioperative systemic oxygen delivery, by means of fluids and inotropes, can be the best way to improve renal perfusion and oxygenation in high-risk patients undergoing major abdominal and orthopedic surgery.
\end{abstract}

Keywords: Postoperative acute renal injury, Perioperative hemodynamic optimization, High-risk patients, Fluid therapy, Oxygen delivery, Cardiac output

\section{Background}

Acute kidney injury (AKI) is an abrupt decrease of renal function, encompassing various etiologies, from prerenal azotemia to acute tubular necrosis and post-renal obstructive disease. More than one condition may coexist in the same patient, making a uniform definition still a challenge. Recently, a single definition was proposed to be useful for practice and research (i.e., an increase of

* Correspondence: mariateresagiglio@gmail.com

Anesthesia and Intensive Care Unit, Department of Emergency and Organ

Transplantation, University of Bari, Piazza G. Cesare, 11, 70124 Bari, Italy serum creatinine of 1.5 to 1.9 times baseline or $\geq 0.3$ $\mathrm{mg} / \mathrm{dl}$, or urine output $<0.5 \mathrm{ml} / \mathrm{kg} / \mathrm{h}$ for 6 to $12 \mathrm{~h} \mathrm{[1]}$.

AKI is a well-known complication following surgical procedures [2], independently associated with increased hospital mortality and doubling of hospital costs [3, 4]. Therefore, the prevention of this postoperative complication is of paramount importance.

Perioperative monitoring and manipulation of physiologic hemodynamic parameters to reach adequate cardiac output $(\mathrm{CO})$ and oxygen delivery $\left(\mathrm{DO}_{2}\right)(\mathrm{GDT})$ may decrease the risk of postoperative renal injury [5]. This finding has been confirmed by a subsequent systematic 
review [6], and in a recent international, web-enabled consensus conference [7], GDT resulted in the strongest recommendation proposed to reduce mortality in patients with or at risk for AKI. Similarly, recent guidelines suggest GDT to prevent the development or worsening of AKI in a perioperative setting (strength of recommendation 2C) [1]. However, interventions to optimize hemodynamics are heterogeneous in targets, timing, design, and technology. Several questions remain unanswered, such as targets, treatment strategiesincluding the role of fluids and inotropes-and kind of patients and surgeries that can benefit from this approach. In order to clarify these issues, an up-to-date systematic review with meta-analysis and trial sequential analysis (TSA) has been performed.

\section{Main body \\ Materials and methods \\ Eligibility criteria}

Studies were searched according to the following eligibility criteria [8]:

Types of participants Adult (age 18 years or over) patients undergoing major surgery were considered. Studies involving mixed population of critically ill, nonsurgical patients, or postoperative patients with already established sepsis or organ failure were excluded.

Type of intervention GDT was defined as perioperative monitoring and manipulation of hemodynamic parameters to reach normal or supranormal values by fluids alone or in combination with inotropic therapy, within $8 \mathrm{~h}$ after surgery. Studies including late hemodynamic optimization treatment were excluded.

Type of comparison Randomized controlled trials (RCTs) comparing the beneficial and harmful effects of GDT and standard hemodynamic therapy were considered. Standard hemodynamic management was defined as anesthesiologists' routine administration of fluids and/or inotropic drugs in order to achieve hemodynamic stability, but not aimed to reach physiologic flow-related end-points and not guided by appropriate monitoring. RCTs with no description or no difference in optimization strategy between groups and RCTs with therapy titrated to the same goal in both groups or not titrated to predefined endpoints were excluded.

Type of outcome measures Primary outcome measure was AKI, whichever definition was adopted. A sensitivity analysis was planned, according to the risk of bias of included studies (i.e., RCTs with 5 or 6 green plus, see below). A TSA was performed including all
RCTs and low risk of bias trials, to adjust for random error risk.

Several subgroup analyses were planned for the main outcome according to:

- Target. Studies were defined according to the target used in the GDT protocol (indices of preload responsiveness, $\mathrm{CO}$ or $\mathrm{DO}_{2}$, or other indirect indices of $\mathrm{DO}_{2}$, such as lactate and venous saturation).

- Treatment. The subset analysis included studies that used fluids alone to optimize hemodynamic status. The other subgroup included studies that used both fluids and inotropes. Moreover, we planned a subgroup analysis including only those RCTs that showed a statistical difference between treatment and control group during the perioperative period in the total amount of starch-based solutions (HES) administered.

- Risk. Studies were split into 2 different subgroups, according to the risk of perioperative morbidity/ mortality. Low risk was defined as elective surgery in young, ASA I-II patients. Definition of high risk was based on the need of emergent surgery, and/or elective major surgery in patients with risk criteria defined by perioperative scoring system [9], ASA physical status classification (i.e., ASA III-IV), age > 60 years, and preoperative morbidity.

- Surgery. Studies were divided according to the kind of surgery (i.e., major abdominal, trauma, vascular, cardiac, thoracic, orthopedic).

Types of studies Randomized controlled trials studying perioperative GDT. No language, publication date, or status restrictions were imposed.

\section{Information sources}

Different search strategies (last update September 2018) were performed to retrieve relevant studies using MEDLINE, The Cochrane Library, and EMBASE databases. No date restriction was applied for MEDLINE and The Cochrane Library databases, while the search was limited to 2007-2018 for EMBASE database [10]. Additional RCTs were searched in The Cochrane Library and in the DARE databases and the reference lists of previously published reviews and retrieved articles, and other data sources were hand-searched in the annual proceedings (2003-2018) of the Society of Critical Care Medicine, the European Society of Intensive Care Medicine, the Society of Cardiovascular Anesthesiologists, the Royal College of Anaesthetists, and the American Society of Anesthesiologists. 


\section{Search}

The search strategies used for MEDLINE, The Cochrane Library, and EMBASE databases are reported in Additional file 1.

\section{Study selection}

Two investigators (MG, NB) examined at first each title and abstract to exclude clearly irrelevant studies and to identify potentially relevant articles. Other two investigators (FP, LD) independently determined the eligibility of full-text articles retrieved. The names of the author, institution, journal of publication, and results were unknown to the two investigators at this time.

\section{Data collection process}

Data were independently collected by two investigators (MG, FP) with any discrepancy resolved by re-inspection of the original article. To avoid transcription errors, the data were input into statistical software and rechecked by different investigators (NB, LD).

\section{Data items}

Data abstraction included patients' characteristics (age, sex), risk factors, type of hemodynamic GDT (monitoring tools, end-points, therapeutic interventions), type of surgery, incidence (patients who developed postoperative AKI), and definition of postoperative AKI.

\section{Risk of bias in individual studies}

A domain-based evaluation, as proposed by the Cochrane Collaboration, was used to evaluate the methodological quality of RCTs [11]. This is a two-part tool, addressing seven specific domains (namely, sequence generation, allocation concealment, blinding of participants and personnel, blinding of outcome assessment, incomplete outcome data, selective outcome reporting, and "other issues") strongly associated with bias reduction $[12,13]$. With regard to blinding, studies in which outcome variables were collected by investigators not aware of the intra-operative strategy, as well as studies in which postoperative renal injury was clearly predefined, were considered adequately masked.

Each domain in the tool includes one or more specific entries in a "risk of bias" table. Within each entry, the first part of the tool describes what was reported to have happened in the study, in sufficient detail to support a judgment about the risk of bias. The second part of the tool assigns a judgment relating to the risk of bias for that entry. This is achieved by assigning a judgment of "low risk," "high risk," or "unclear risk" of bias. After each domain is completed, a "risk of bias summary" figure presenting all of the judgments in a cross-tabulation of study by entry is generated. The green plus indicates a low risk of bias, the red minus indicates a high risk of bias, and the white color indicates an unclear risk of bias. For each study, the number of green plus obtained for every domain was calculated: RCTs with 5 or 6 green plus were considered as low risk of bias studies.

\section{Summary measures and planned method of analysis}

Meta-analytic techniques (analysis software RevMan, version 5.3 Cochrane Collaboration, Oxford, England, UK) were used to combine studies using odds ratios (OR) and 95\% confidence intervals (CI). A statistical difference between groups was considered to occur if the pooled 95\% CI did not include 1 for the OR. An OR less than 1 favored GDT when compared with the control group. Two-sided $p$ values were calculated. A randomeffects model was chosen for all analyses. Statistical heterogeneity and inconsistency were assessed by using the $Q$ and $I^{2}$ tests, respectively $[14,15]$. When the $p$ value of the $Q$ test was $<0.10$ and/or the $I^{2}$ was $>40 \%$, heterogeneity and inconsistency were considered significant [16]. When significant heterogeneity and inconsistency were found, the most heterogeneous study on the basis of the forest plot was removed and the analysis was redone. Two TSA were performed including all trials and only low risk of bias RCTs. The information size and monitoring boundary were calculated anticipating a $2 \%$ relative risk reduction in postoperative AKI with GDT. We set risk of type I at 5\% and power at $95 \%$.

\section{Results}

\section{Study selection}

The search strategies identified 3304 (MEDLINE), 9992 (Cochrane Library), and 3492 (EMBASE) articles. Fifteen articles were identified through other sources (congress abstracts, reference lists). After initial screening and subsequent selection, a pool of 126 potentially relevant RCTs was identified. The subsequent eligibility process (Additional file 2: Figure S1) excluded 61 articles and, therefore, 65 [17-81] RCTs with a total sample of 9308 patients were considered for the analysis.

\section{Study characteristics}

All included articles evaluated the effects of hemodynamic GDT on morbidity (including AKI) as primary or secondary outcome and had a population sample of adult surgical patients, undergoing both elective and emergent procedures (Additional file 7: Table S1). The studies were performed in Australia, USA, Europe, Canada, Brazil, China, Africa, and India from 1991 to 2018 and were all published in English.

Data concerning morbidity/mortality risk definition, population, type of surgery, monitoring tools, and targets are presented in Additional file 7: Table S1. The risk of bias assessment for each trial is showed in Additional file 8: Table S2. Out of 65 studies, 29 


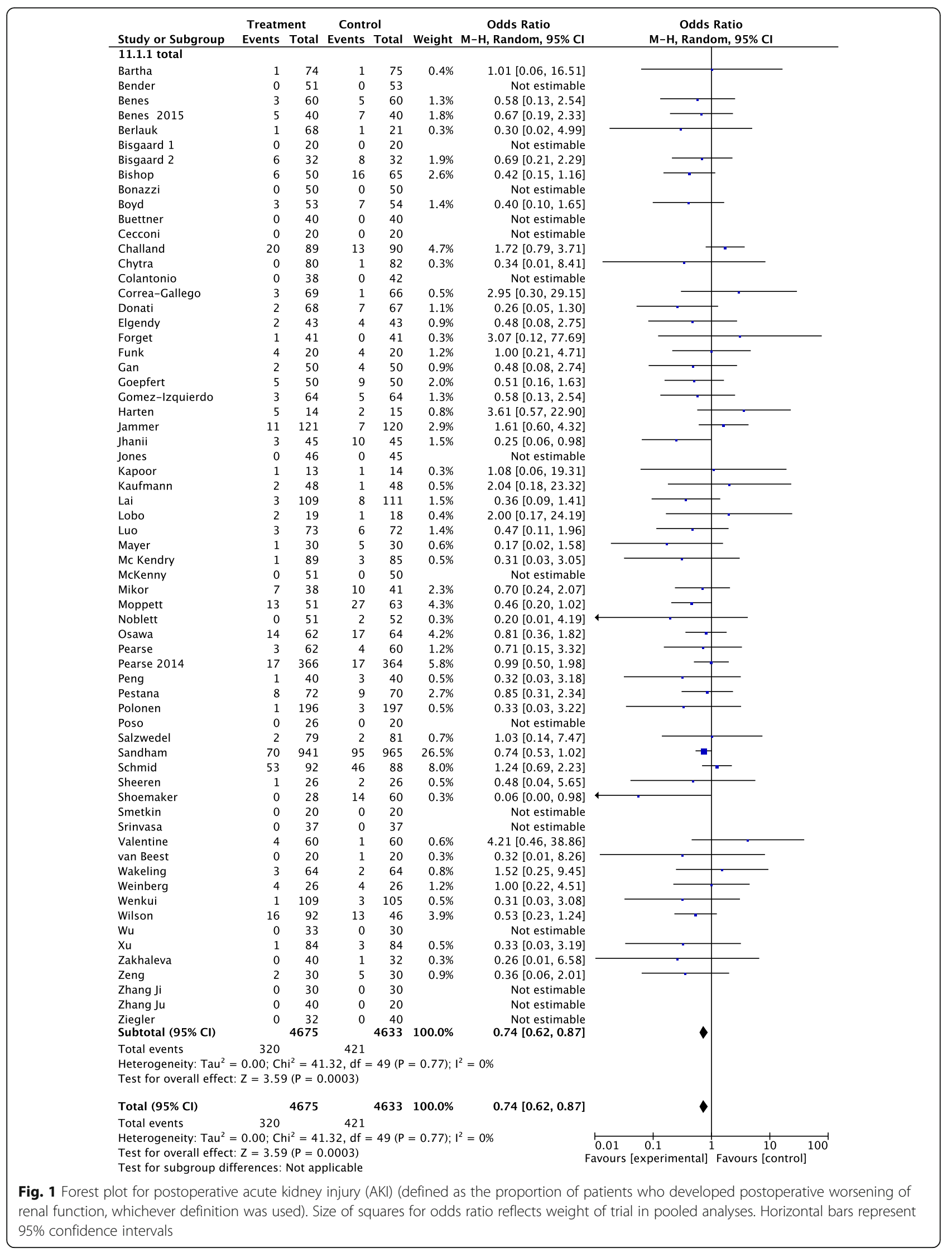




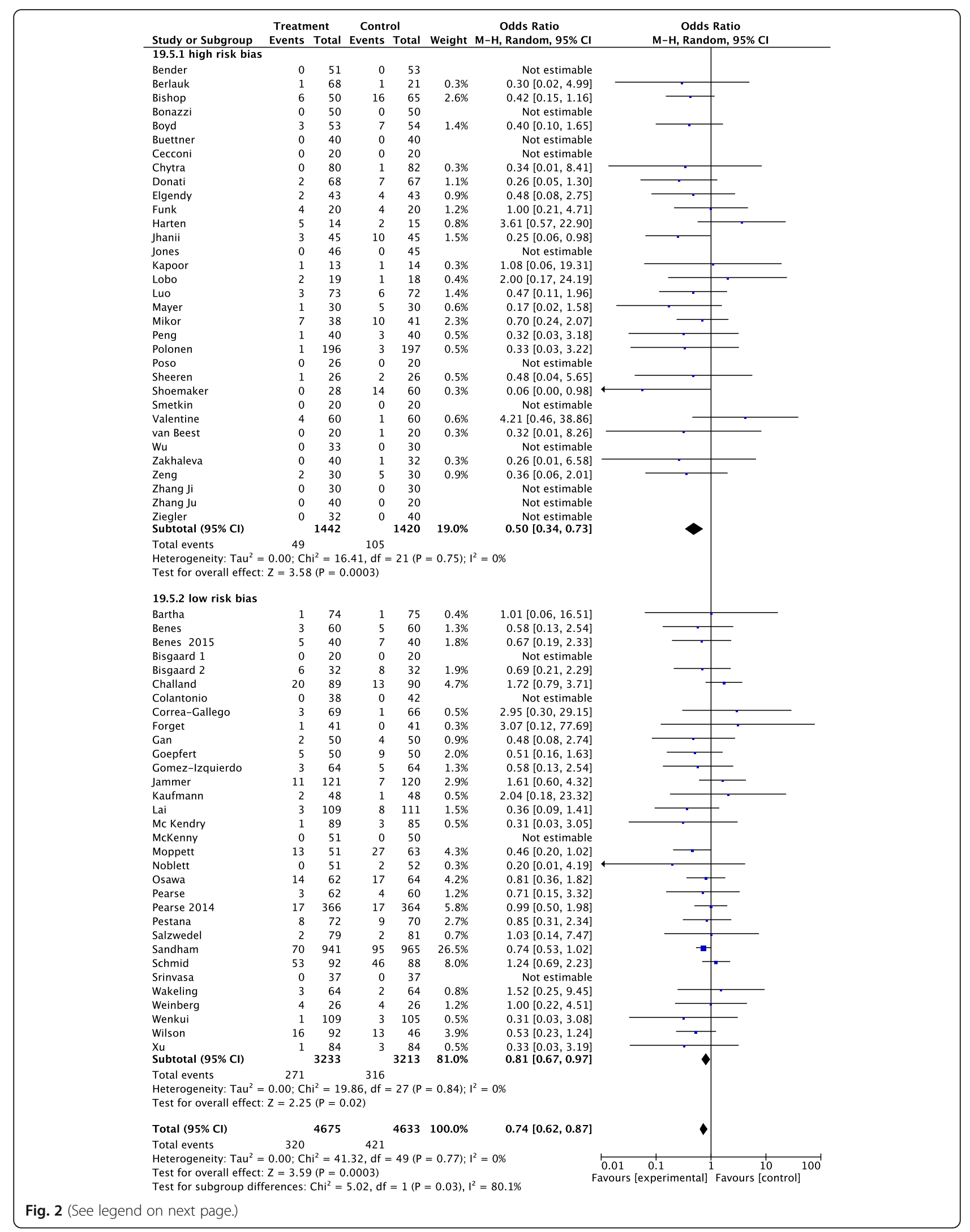


(See figure on previous page.)

Fig. 2 Forest plot for postoperative acute kidney injury (AKI) (defined as the proportion of patients who developed postoperative worsening of renal function, whichever definition was used by the authors of the included studies). Studies were split in high and low risk of bias, according to a domain-based evaluation, as proposed by the Cochrane Collaboration. RCTs with 5 or 6 green plus were considered as having an overall low risk of bias (see text for details). Size of squares for odds ratio reflects weight of trial in pooled analyses. Horizontal bars represent $95 \%$ confidence intervals

enrolled "high-risk" patients. In 26 studies, the treatment group received only fluids (crystalloids, gelofusine, HES) and/or blood, while in 39 studies, optimization was obtained both with fluids (crystalloids and/or colloids and/or blood) and inotropes (dopamine, dobutamine, dopexamine, or epinephrine) with vasodilators. In one study [74], either dopexamine or epinephrine was administered in the treatment group; both groups were pooled together for the purpose of the analysis. In one study [42], one treatment group received dopamine not targeted to hemodynamic end-point, and therefore, this group was not considered for the purpose of the analysis.

\section{Quantitative data synthesis}

Among the 9308 patients randomized in the 65 included studies, 741 developed AKI. Of these, 421 had been randomized to control group (9.01\%) and 320 (6.84\%) to GDT. The pooled OR and $95 \%$ CI for the development of AKI were 0.64, 95\% CI 0.62-0.87; $p=0.0003$ (Fig. 1). No statistical heterogeneity was detected.

TSA confirmed the main result: the cumulative $z$ curve crosses the O'Brien-Fleming boundaries, and the meta-analysis can be declared as conclusive with regard to the effect of GDT on AKI (Additional file 3: Figure S2). The sensitivity analyses, including only low risk of bias RCTs, confirmed the main analysis, with an OR 0.81, 95\% CI 0.6-0.97 (Fig. 2). TSA considering only low risk of bias RCTs shows the same conclusion: the cumulative $z$ curve crossed the O'Brien-Fleming boundaries, indicating firm evidence that GDT reduces postoperative AKI (Additional file 4: Figure S3).

The subset analysis including studies using $\mathrm{CO}$ or $\mathrm{DO}_{2}$ as hemodynamic target showed a significant reduction in AKI (OR 0.64, 95\% CI 0.62 to $0.89, p=0.001$ ), while OR of studies that used preload indices or indirect indices as lactate did not reach statistical significance (Fig. 3).

Subgroup analysis showed that fluid administration alone did not reduce AKI, while a significant decrease in AKI rate was observed in patients receiving both fluids and inotropes (OR $0.7395 \%$ CI 0.60 to $0.89, p=0.001$ ) (Fig. 4). The subgroup analysis including RCTs that reported a statistical difference between treatment and control group in total amount of HES given during the perioperative period showed no difference in postoperative AKI (OR $0.8595 \%$ CI 0.64 to $1.12, p=0.24 ; I^{2} 0 \%$, 33 RCTs, 3871 patients) (Additional file 5: Figure S4).
Postoperative AKI rate was significantly lower in studies enrolling "high-risk" patients (OR, 0.72; 95\% CI 0.59 to $0.87, p=0.0008$ ); in low-risk patients, no difference in AKI was observed (Fig. 5).

Subgroup analyses based on the kind of surgery yielded significant differences in AKI between treatment and control groups for patients undergoing major abdominal and orthopedic surgery (OR 0.78; 95\% CI 0.63$0.96, p=0.03$, and OR $0.51 ; 95 \%$ CI $0.27-0.96, p=0.04$, respectively), while no difference was observed in other types of surgical procedures (Additional file 6: Figure S5) . Excluding the heaviest trial [63] did not significantly change main and subgroup analyses, while the sensitivity analysis, including only low risk of bias RCTs, yielded a not significant reduction in AKI.

\section{Discussion}

The present meta-analysis demonstrates that the incidence of postoperative AKI is reduced by GDT: this significant reduction was confirmed in the sensitivity analysis enrolling only low risk of bias trials. TSA, performed to unmask false-positive results [82], confirms the robustness of the data, since the number of patients enrolled (9308 patients) is very near to the required information size (9668 patients) to reach a definite conclusion. In order to reduce AKI incidence, a strategy that is guided by $\mathrm{CO}$ and $\mathrm{DO}_{2}$ should be adopted, using both fluids and inotropes. Patients who more benefit from this approach are high-risk patients undergoing abdominal or orthopedic surgery.

The role of kidney hypoperfusion and hypoxia has been recently underlined as a key pathogenetic event promoting postoperative AKI [83]. Tissue hypoxia triggers a vicious cycle of inflammation, peritubular capillary narrowing, impaired renal autoregulation, oxidative stress, apoptosis, and necrosis [84]. Protection against hypoperfusion mainly relies on maintaining adequate intravascular volume and organ perfusion pressure. Several evidence confirm this approach, and a very recent trial [85] suggests that an "individualized" blood pressure control, with a protocolized hemodynamic algorithm to guide fluid delivery and to maximize stroke volume, could reduce the incidence of AKI.

Other authors [86] confirmed that intraoperative lactic acidosis or vasopressor requirement precedes subsequent AKI development and that failure to achieve preoperative $\mathrm{DO}_{2}$ is significantly associated with the increase of postoperative creatinine. Interestingly, AKI 


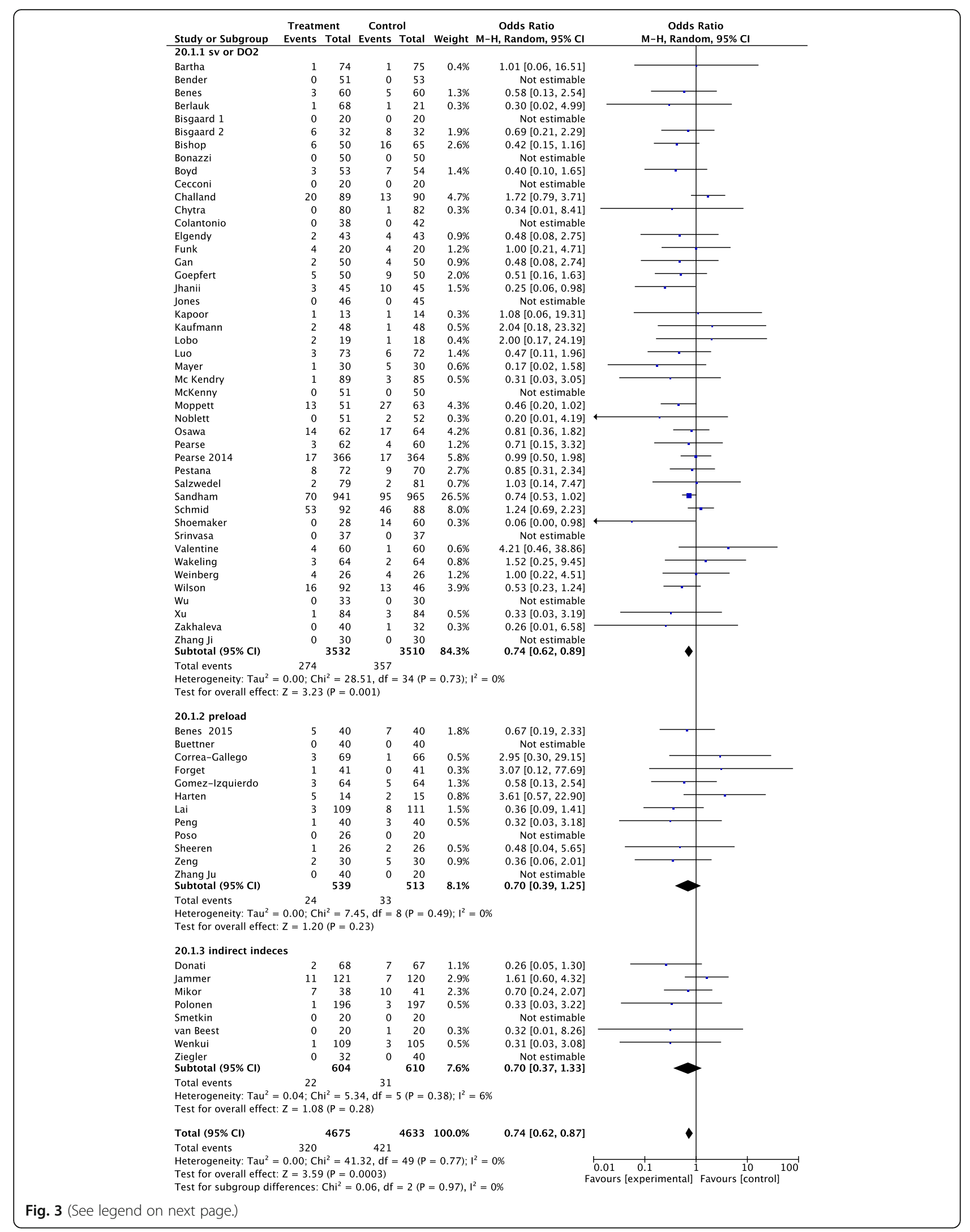


(See figure on previous page.)

Fig. 3 Forest plot for postoperative acute kidney injury (AKI) (defined as the proportion of patients who developed postoperative worsening of renal function, whichever definition was used). Studies were defined according to the target used in the GDT protocol (stroke volume and oxygen delivery or other indexes of oxygen delivery, indices of fluid-responsiveness, such as pulse pressure variation or stroke volume variation, and mixed venous oxygen saturation or other indirect indexes of oxygen delivery such as lactate). Size of squares for odds ratio reflects weight of trial in pooled analyses. Horizontal bars represent 95\% confidence intervals

was not prevented by GDT or standard care after lactic acidosis developed or vasopressors were required. Taking together, these data suggest that both lactic acidosis and hypotension may be late indicators of a reduction of renal perfusion pressure, and that, in order to avoid $\mathrm{AKI}$, the best goal is to maintain an individualized $\mathrm{DO}_{2}$. Basing on this rationale, fluid resuscitation is crucial to maintain $\mathrm{CO}$ and renal blood flow. GDT allows a timelier fluid replacement strategy in patients who need it, avoiding at the same time excessive fluid loading in patients that do not [5]. In all GDT protocols, however, fluid resuscitation is only the first step. In patients who cannot achieve adequate $\mathrm{DO}_{2}$, inotropes are necessary, acting in a synergistic manner with fluids, since GDT fluid therapy allows optimal use of inotropic drugs, and inotropic drugs reduce the risk of fluid overload, optimizing $\mathrm{CO}$ [5]. Our results further reinforce these figures, since GDT guided by $\mathrm{CO}$ and $\mathrm{DO}_{2}$ as hemodynamic target, with fluids and inotropes, shows a significant reduction in AKI.

Recent evidence suggests that the type of fluid may be critical in determining AKI [87]. Several concerns about renal toxicity of HES solutions have been raised, and their safety in surgical patients is still under debate [88, 89]. We tried to investigate the effect of GDT adopting HES solutions: the subgroup analysis including only RCTs that showed a statistical difference between treatment and control group during the perioperative period in the total amount of HES administered did not find any statistical difference in AKI incidence. In most studies, HES were used both in intervention and control groups. Therefore, in order to explore the association between AKI and HES, we tried to select papers on the basis of a significant difference in the amount given. Colloids seem neither to benefit nor to harm AKI if given within an individualized, timely fluid "replacement" strategy. Interestingly, a very recent RCT [90] reached the same results. However, no clear conclusion can be drawn, since, paradoxically, colloids might harm the kidney in the context of a beneficial GDT effect. Further trials are needed to investigate the effect of starch solution on AKI in surgical patients.

GDT significantly reduced the incidence of postoperative AKI in high-risk patients that included aged people, ASA III-IV, with increased risk of mortality and morbidity due to reduced cardiovascular reserve, undergoing high-risk procedures with increased risk of blood loss and/or fluid shift. These characteristics are all wellknown risk factors for postoperative renal injury [91]. Therefore, it is logical to argue that this category of frail patients would more benefit from GDT to improve systemic oxygenation and to maintain organ perfusion.

The subgroup analysis on surgeries showed that GDT significantly reduced AKI after abdominal and orthopedic procedures, while no effect was seen in other surgeries. Surgical stress may increase oxygen demand up to $40 \%$ in major abdominal surgery [92]. Moreover, major abdominal surgery can cause an increase of intraabdominal pressure, linked to an increase in capillary permeability and interstitial fluid accumulation or to a diminished abdominal wall compliance that, in turn, causes intrarenal vascular congestion with a reduction in renal perfusion [83]. On the other side, orthopedic patients include often very aged people with severe comorbidities (i.e., hypertension, renal failure, diabetes) that easily expose them to an increased risk of postoperative AKI [93]. Recent findings suggest that advanced age, hypertension, general anesthesia, and low intraoperative arterial pressure are all risk factors for AKI after joint replacement surgery [94]. Therefore, a strategy aimed to maintain $\mathrm{CO}$ seems reasonable to protect against AKI, at least in these surgical settings. No definite conclusion on other surgeries could be drawn, since the low number of included trials in other subgroup analysis is not sufficient to detect any effect, precluding any definite conclusions.

This study has a number of limitations. No attempt was made to correct for the type or quantity of fluids or inotropes given, because they are inconsistently reported in the literature and have a demonstrable wide variability in their dosing across studies. Moreover, the included studies vary in terms of hemodynamic monitoring, the goals, and the timing of intervention: this could have introduced a relatively high clinical heterogeneity, although the results remain consistent across a number of subgroups and sensitivity analyses.

Additional well-designed RCTs are necessary to reach the target of an "individualized" GDT, for example by better defining renal risk, or preoperative cardiological performance, the amount of fluid, and the dose of vasoactive administered, using accepted and uniform definitions, as well as a consistent AKI definition, like KIDGO proposes. Recent trials $[95,96]$ gave some interesting insight on this approach, suggesting that an implementation of the 


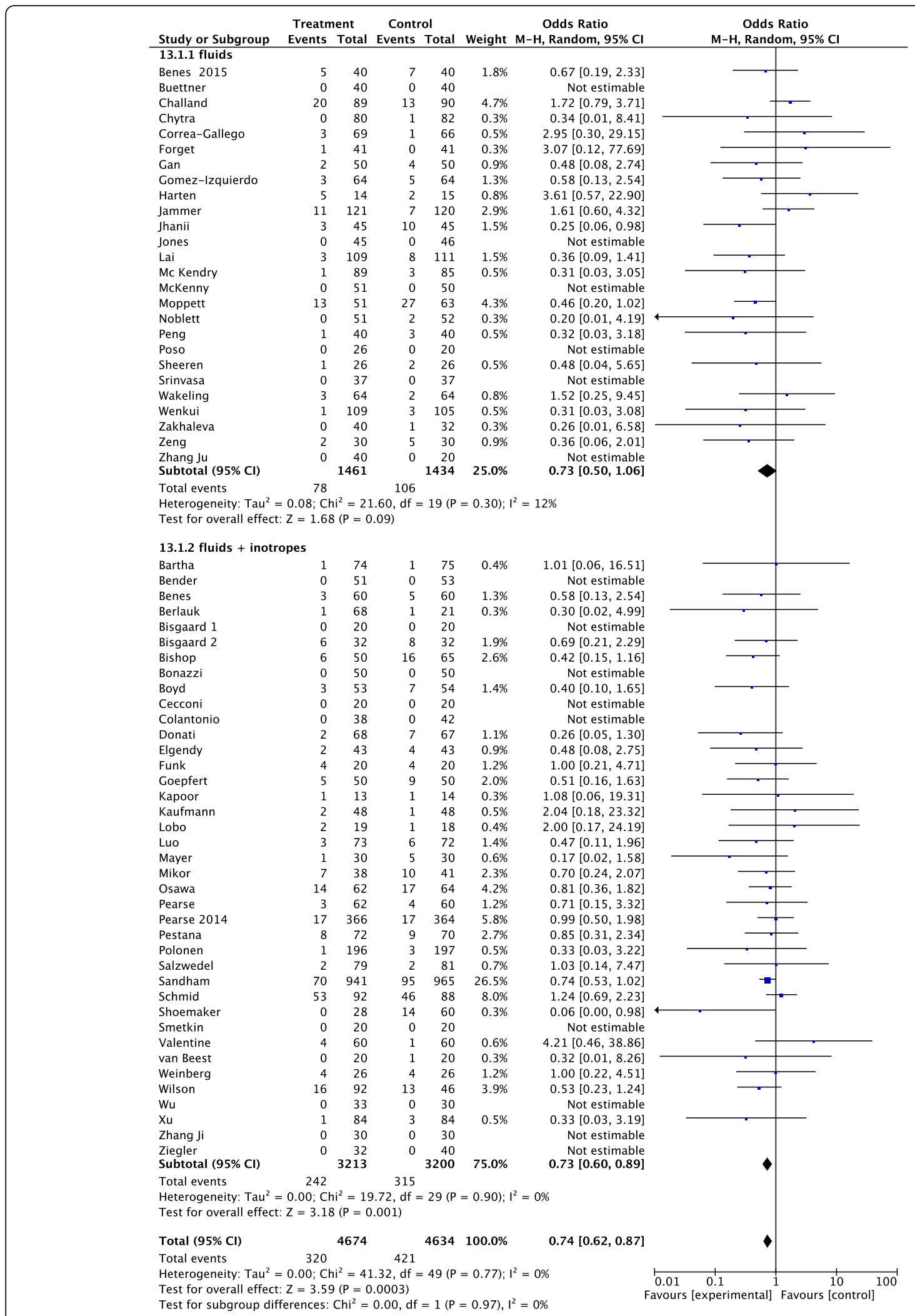

Fig. 4 Forest plot for postoperative acute kidney injury (AKI) (defined as the proportion of patients who developed postoperative worsening of renal function, whichever definition was used). Studies were split into trials that used fluids alone or both fluids and inotropes. Size of squares for odds ratio reflects weight of trial in pooled analyses. Horizontal bars represent 95\% confidence intervals 


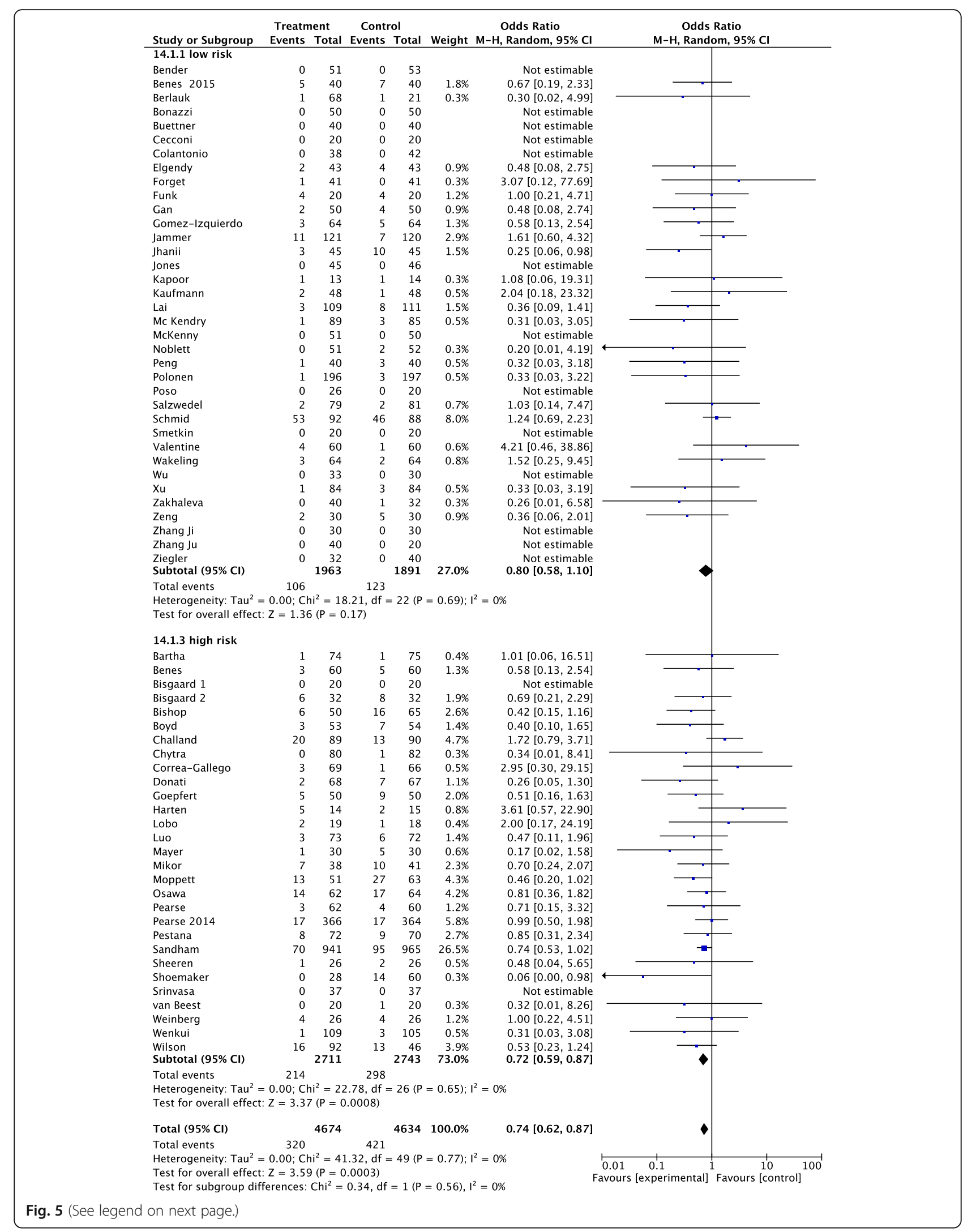


(See figure on previous page.)

Fig. 5 Forest plot for postoperative acute kidney injury (AKI) (defined as the proportion of patients who developed postoperative worsening of renal function, whichever definition was used by the authors of the included studies). Studies were split into 2 different subgroups, according to the risk of perioperative morbidity/mortality. Low risk was defined as elective surgery in young, ASA I-II patients. Definition of high risk was based on the need of emergent surgery, and/or elective major surgery in patients with risk criteria defined by perioperative scoring system, ASA physical status classification (i.e., ASA III-IV), age > 60 years, and preoperative morbidity. Size of squares for odds ratio reflects weight of trial in pooled analyses. Horizontal bars represent 95\% confidence intervals

KDIGO guidelines, including hemodynamic optimization, reduced the frequency and severity of postoperative AKI in high-risk patients, identified by urinary biomarkers.

\section{Conclusions}

This up-to-date meta-analysis, within the limitations of existing data, the high clinical heterogeneity and the analytic approaches used, confirms that GDT significantly reduces postoperative AKI. The result is reinforced by TSA and considering only low risk of bias trials. Moreover, it suggests that targeting GDT to perioperative systemic $\mathrm{DO}_{2}$, by means of fluid and inotropes, is the simplest way to improve renal perfusion, at least in high-risk patients undergoing abdominal or orthopedic procedures.

\section{Additional files}

Additional file 1: Search strategies. (DOCX $9 \mathrm{~kb}$ )

Additional file 2: Figure S1. Flow chart summarizing the studies selection procedure for the meta-analysis. (TIFF $1519 \mathrm{~kb}$ )

Additional file 3: Figure S2. Trial sequential analysis of postoperative acute kidney injury, including all trials. A diversity adjusted information size of 9668 patients was calculated using $\alpha=0.05$ (two-sided), $\beta=0.20$ (power 95\%), an anticipated relative risk reduction of $2 \%$, and an event proportion of $9 \%$ in the control arm. The blue cumulative $z$ curve was constructed using a random effects model. (TIFF $3072 \mathrm{~kb}$ )

Additional file 4: Figure S3. Trial sequential analysis of postoperative acute kidney injury, including only low risk of bias trials. A diversity adjusted information size of 9668 patients was calculated using $a=0.05$ (two-sided), $\beta=0.20$ (power 95\%), an anticipated relative risk reduction of $2 \%$, and an event proportion of $9 \%$ in the control arm. The blue cumulative $z$ curve was constructed using a random effects model. (TIFF $3072 \mathrm{~kb}$ )

Additional file 5: Figure S4. Forest plot for postoperative acute kidney injury (AKI) (defined as the proportion of patients who developed postoperative worsening of renal function, whichever definition was used) including only those RCTs that showed a statistical difference between treatment versus control group during the perioperative period in the total amount of starch-based solutions (HES) administered. Size of squares for odds ratio reflects weight of trial in pooled analyses. Horizontal bars represent 95\% confidence intervals. (TIFF 3072 kb)

Additional file 6: Figure S5. Forest plot for postoperative acute kidney injury (AKI) (defined as the proportion of patients who developed postoperative worsening of renal function, whichever definition was used). Studies were divided according to the kind of surgery (i.e., major abdominal, vascular, cardiac, thoracic, orthopedic, trauma surgery). Size of squares for odds ratio reflects weight of trial in pooled analyses. Horizontal bars represent 95\% confidence intervals. (EPS $1847 \mathrm{~kb}$ )

Additional file 7: Table S1. Data concerning RCTs morbidity/mortality risk definition, population and type of surgery, tools and target used. (DOCX 23 kb)

Additional file 8: Table S2. The risk of bias assessment for each trial, according to the Cochrane domain-based evaluation. (DOCX $17 \mathrm{~kb}$ )

\section{Abbreviations}

AKI: Acute kidney injury; ASA: American Society of Anesthesiologists; $\mathrm{Cl}$ : Confidence intervals; $\mathrm{CO}$ : Cardiac output; $\mathrm{DO}_{2}$ : Oxygen delivery; GDT: Goal-directed therapy; HES: Starch-based solutions; OR: Odds ratios; RCTs: Randomized controlled trials; TSA: Trial sequential analysis

\section{Authors' contributions}

All authors take responsibility for the integrity of the work as a whole. All authors have contributed to the conception, design, and acquisition of data and analysis and interpretation of data (see text for details). All authors have participated in drafting the article, revising it, and approving the final version to be published.

\section{Funding}

No funding, except for department's sources.

\section{Availability of data and materials}

All data generated or analyzed during this study are included in this published article [and its supplementary information files].

Ethics approval and consent to participate Not applicable.

\section{Consent for publication}

Not applicable.

\section{Competing interests}

The authors declare that they have no competing interests.

Received: 22 February 2019 Accepted: 13 June 2019

Published online: 26 June 2019

\section{References}

1. Kellum J, Lameire N, for the KDIGO AKI Guideline Work Group. Diagnosis, evaluation, and management of acute kidney injury: a KDIGO summary (part 1). Crit Care. 2013;17:204.

2. Zarbock A, Koyner JL, Hoste EAJ, et al. Update on perioperative acute kidney injury. Anesth Analg. 2018;127(5):1236-45.

3. Deng Y, Yuan J, Chi R, et al. The incidence, risk factors and outcomes of postoperative acute kidney injury in neurosurgical critically ill patients. Sci Rep. 2017;7(1):4245.

4. Long TE, Helgason D, Helgadottir S, et al. Acute kidney injury after abdominal surgery: incidence, risk factors, and outcome. Anesth Analg. 2016;122(6):1912-20

5. Brienza N, Giglio MT, Marucci M, et al. Does perioperative hemodynamic optimization protect renal function in surgical patients? A meta-analytic study. Crit Care Med. 2009;37(6):2079-90.

6. Prowle JR, Chua HR, Bagshaw SM, et al. Clinical review: volume of fluid resuscitation and the incidence of acute kidney injury - a systematic review. Crit Care. 2012;16(4):230.

7. Landoni G, Bove T, Székely A, et al. Reducing mortality in acute kidney injury patients: systematic review and international web-based survey. J Cardiothorac Vasc Anesth. 2013;27(6):1384-98.

8. Moher D, Liberati A, Tetzlaff J, et al. Preferred reporting items for systematic reviews and meta-analyses: the PRISMA statement. BMJ. 2009;339:b2535.

9. Copeland GP, Jones D, Walters M. POSSUM: a scoring system for surgical audit. Br J Surg. 1991;78:355-60.

10. Lefebvre C, Manheimer E, Glanville J. Chapter 6: Searching for studies. In: Higgins JPT, Green S (editors). Cochrane Handbook for Systematic Reviews of InterventionsVersion 5.1.0 (updated March 2011). The Cochrane Collaboration; 2011. https://training.cochrane.org/handbook. 
11. Higgins JPT, Altman DG, Sterne JAC (editors). Chapter 8: Assessing risk of bias in included studies. In: Higgins JPT, Green S (editors). Cochrane Handbook for Systematic Reviews of Interventions Version 5.1.0 (updated March 2011). The Cochrane Collaboration; 2011. https://training.cochrane. org/handbook.

12. Jadad AR, Moore RA, Carroll D, et al. Assessing the quality of reports of randomized clinical trials: is blinding necessary? Control Clin Trials. 1996; 17(1):1-12.

13. Jüni $P$, Witschi $A$, Bloch $R$, et al. The hazards of scoring the quality of clinical trials for meta-analysis. JAMA. 1999;282(11):1054-60.

14. Higgins JPT, Thompson SG. Quantifying heterogeneity in a meta-analysis. Stat Med. 2002;21(11):1539-58.

15. Higgins JPT, Thompson SG, Deeks JJ, et al. Measuring inconsistency in meta-analyses. BMJ. 2003;327(7414):557-60.

16. Higgins JPT, Altman DG, Sterne JAC. JPT H, Green S, editors. Cochrane Handbook for Systematic Reviews of Interventions Version 5.1.0 [updated March 2011]. [updated 2011]; cited Available from: https://training.cochrane. org/handbook.

17. Bartha E, Arfwedson C, Imnell A, et al. Randomized controlled trial of goaldirected haemodynamic treatment in patients with proximal femoral fracture. Br J Anaesth. 2013 Apr;110(4):545-53.

18. Bender JS, Smith-Meek MA, et al. Routine pulmonary artery catheterization dos not reduce morbidity and mortality of elective vascular surgery: results of a prospective, randomized trial. Ann Surg. 1997;226(3):229-36.

19. Benes J, Chytra I, Altmann P, et al. Intraoperative fluid optimization using stroke volume variation in high risk surgical patients: results of prospective randomized study. Crit Care. 2010;14(3):R118.

20. Benes J, Haidingerova L, Pouska J, et al. Fluid management guided by a continuous non-invasive arterial pressure device is associated with decreased postoperative morbidity after total knee and hip replacement. BMC Anesthesiol. 2015;15:148.

21. Berlauk JF, Abrams JH, Gilmour IJ, et al. Preoperative optimization of cardiovascular hemodynamics improves outcome in peripheral vascular surgery: a prospective, randomized clinical trial. Ann Surg. 1991;214:289-99.

22. Bisgaard J, Gilsaa T. Rønholm E, et al Haemodynamic optimisation in lower limb arterial surgery: room for improvement? Acta Anaesthesiol Scand. 2013;57(2):189-98.

23. Bisgaard J, Gilsaa T, Rønholm E, et al. Optimising stroke volume and oxygen delivery in abdominal aortic surgery: a randomised controlled trial. Acta Anaesthesiol Scand. 2013;57(2):178-88.

24. Bishop MH, Shoemaker WC, Appel $P$, et al. Prospective, randomized trial of survivor values of cardiac index, oxygen delivery, and oxygen consumption as resuscitation endpoints in severe trauma. J Trauma. 1995;38(5):780-7.

25. Bonazzi M, Gentile F, Biasi GM, et al. Impact of perioperative haemodynamic monitoring on cardiac morbidity after major vascular surgery in low risk patients. A randomised pilot trial. Eur J Vasc Endovasc Surg. 2002;23(5):445-51.

26. Boyd O, Grounds M, Bennett D. A randomized clinical trial of the effect of deliberate perioperative increase of oxygen delivery on mortality in highrisk surgical patients. JAMA. 1993;270:2699-708.

27. Buettner M, Schummer W, Huettemann E, et al. Influence of systolicpressure-variation-guided intraoperative fluid management on organ function and oxygen transport. Br J Anaesth. 2008 Aug;101(2):194-9.

28. Cecconi M, Fasano N, Langiano N, et al. Goal-directed haemodynamic therapy during elective total hip arthroplasty under regional anaesthesia. Crit Care. 2011;15(3):R132.

29. Challand C, Struthers R, Sneyd JR, et al. Randomized controlled trial of intraoperative goal-directed fluid therapy in aerobically fit and unfit patients having major colorectal surgery. Br J Anaesth. 2012 Jan;108(1):53-62.

30. Chytra I, Pradl R, Bosman R, et al. Esophageal Doppler-guided fluid management decreases blood lactate levels in multiple-trauma patients: a randomized controlled trial. Crit Care. 2007;11(1):R24

31. Colantonio L, Claroni C, Fabrizi L, et al. A randomized trial of goal directed vs standard fluid therapy in cytoreductive surgery with hyperthemic intraperitoneal chemotherapy. J Gastrointest Surg. 2015;19(4):722-9.

32. Correa-Gallego C, See Tan K, Arslan-Carlon V, et al. Goal-directed fluid therapy using stroke volume variation for resuscitation after low central pressure-assisted liver resection: a randomized clinical trial. J Am Coll Surg. 2015;221(2):591-601

33. Donati A, Loggi S, Preiser JC, et al. Goal-directed intraoperative therapy reduces morbidity and length of hospital stay in high-risk surgical patients. Chest. 2007;132:1817-24.
34. Elgendy MA, Esmat IM, Kassim DY. Outcome of intraoperative goal-directed therapy using Vigileo/FloTrac in high-risk patients scheduled for major abdominal surgeries: a prospective randomized trial. Egypt J Anaesth. 2017; 33:263-9.

35. Forget $P$, Lois F, de Kock M. Goal-directed fluid management based on the pulse oximeter derived pleth variability index reduces lactate levels and improves fluid management. Anesth Analg. 2010;111(4):910-4.

36. Funk D, HayGlass K, Koulak J, et al. A randomized controlled trial on the effects of goal-directed therapy on the inflammatory response open abdominal aortic repair. Crit Care. 2015;19:247.

37. Gan TJ, Soppitt A, Maroof M, et al. Goal-directed intraoperative fluid administration reduces length of hospital stay after major surgery. Anesthesiology. 2002;97:820-6.

38. Goepfert MS, Richter HP, Eulenburg CZ, et al. Individually optimized hemodynamic therapy reduces complications and length of stay in the intensive care unit: a prospective, randomized controlled trial. Anesthesiology. 2013;119(4):824-36.

39. Gómez-Izquierdo JC, Trainito A, Mirzakandov D, et al. Goal-directed fluid therapy does not reduce primary postoperative ileus after elective laparoscopic colorectal surgery: a randomized controlled trial. Anesthesiology. 2017;127(1):36-49.

40. Harten J, Crozier JEM, MCCreath B, et al. Effect of intraoperative fluid optimisation on renal function in patients undergoing emergency abdominal surgery: a randomised controlled pilot study. Int J Surg. 2008; 6(3):197-204.

41. Jammer I, Ulvik A, Erichsen C, et al. Does central venous oxygen saturationdirected fluid therapy affect postoperative morbidity after colorectal surgery? A randomized assessor-blinded controlled trial. Anesthesiology. 2010;113(5):1072-80.

42. Jhanji S, Vivian-Smith A, Lucena-Amaro S, et al. Haemodynamic optimisation improves tissue microvascular flow and oxygenation after major surgery: a randomised controlled trial. Crit Care. 2010;14(4):R151. https://doi.org/10. 1186/cc9220.

43. Jones $C$, Kelliher $L$, Dickinson M, et al. Randomized clinical trial on enhanced recovery versus standard care following open liver resection. $\mathrm{Br}$ J Surg. 2013;100(8):1015-24.

44. Kapoor M, Kakani M, Chowdhury U, et al. Early goal-directed therapy in moderate to high-riosk cardiac surgery patients. Ann Card Anaesth. 2008;11:27-34.

45. Kaufmann KB, Stein L, Bogatyreva L, et al. Oesophageal Doppler guided goal-directed haemodynamic therapy in thoracic surgery - a single centre randomized parallel-arm trial. Br J Anaesth. 2017;118(6):852-61.

46. Lai CW, Starkie T, Creanor S, et al. Randomized controlled trial of stroke volume optimization during elective major abdominal surgery in patients stratified by aerobic fitness. Br J Anaesth. 2015;115(4):578-89.

47. Lobo SM, Salgado PF, Castillo VG, et al. Effects of maximizing oxygen delivery on morbidity and mortality in high-risk surgical patients. Crit Care Med. 2000;28(10):3396-404.

48. Luo J, Xue J, Liu J, et al. Goal-directed fluid restriction during brain surgery: a prospective randomized controlled trial. Ann Intensive Care. 2017;7(1):16.

49. Mayer J, Boldt J, Mengistu A, et al. Goal-directed intraoperative therapy based on autocalibrated arterial pressure waveform analysis reduces hospital stay in high-risk surgical patients: a randomized, controlled trial. Crit Care. 2010;14(10):R18.

50. McKendry M, McGloin H, Saberi D, et al. Randomised controlled trial assessing the impact of a nurse delivered, flow monitored protocol for optimisation of circulatory status after cardiac surgery. BMJ. 2004;329:258.

51. McKenny $M$, Conroy $P$, Wong $A$, et al. Randomised prospective trial of intraoperative oesophageal Doppler-guided fluid administration in major gynaecological surgery. Anaesthesia. 2013;68(12):1224-31.

52. Mikor A, Trásy D, Németh MF, et al. Continuous central venous oxygen saturation assisted intraoperative hemodynamic management during major abdominal surgery: a randomized, controlled trial. BMC Anesthesiol. 2015 Jun 4;15:82.

53. Moppett IK, Rowlands M, Mannings A, et al. LiDCO-based fluid management in patients undergoing hip fracture surgery under spinal anaesthesia: a randomized trial and systematic review. Br J Anaesth. 2014;114(3):444-59.

54. Noblett SE, Snowden CP, Shenton BK, et al. Randomized clinical trial assessing the effect of Doppler-optimized fluid management on outcome after elective colorectal resection. Br J Surg. 2006;93:1069-76.

55. Osawa EA, Rhodes A, Landoni G, et al. Effect of perioperative goal-directed hemodynamic resuscitation therapy on outcomes following cardiac surgery: 
a randomized clinical trial and systematic review. Crit Care Med. 2016;44(4): 724-33.

56. Pearse R, Dawson D, Fawcett J, et al. Early goal-directed therapy after major surgery reduces complications and duration of hospital stay. A randomised, controlled trial [ISRCTN38797445]. Crit Care. 2005;9:687-93.

57. Pearse R, Harrison DA, MacDonald N, et al. Effect of a perioperative, cardiac output-guided haemodynamic therapy algorithm on outcomes following major gastrointestinal surgery: a randomized clinical trial and systematic review. JAMA. 2014;311(21):2181-90.

58. Peng K, Li J, Cheng H, et al. Goal-directed fluid therapy based on stroke volume variations improves fluid management and gastrointestinal perfusion in patients undergoing major orthopedic surgery. Med Princ Pract. 2014;23:413-20.

59. Pestana D, Espinoza E, Eden A, et al. Perioperative goal-directed haemodynamic optimization using noninvasive cardiac output monitoring in major abdominal surgery: a prospective, randomized, multicenter, pragmatic trial: POEMAS study (PeriOperative goal-directed thErapy in Major Abdominal Surgery). Anesth Analg. 2014;119(3):579-87.

60. Polonen $\mathrm{P}$, Ruokonen $\mathrm{E}$, Hippelainen $\mathrm{M}$, et al. A prospective, randomized study of goal-oriented hemodynamic therapy in cardiac surgical patients. Anesth Analg. 2000;90:1052-9.

61. Poso T, Winso O, Aroch W, et al. Perioperative fluid guidance with tranthoracic ecocardiography and pulse contour device in morbidly obese patients. Surg. 2014;21:2117-25.

62. Salzwedel C, Puig J, Carstens A, et al. Perioperative goal-directed hemodynamic therapy based on radial arterial pulse pressure variation and continuous cardiac index trending reduces postoperative complications after major abdominal surgery: a multi-center, prospective, randomized study. Crit Care. 2013;17(5):R191.

63. Sandham JD, Hull RD, Brant RF, et al. A randomized, controlled trial of the use of pulmonary-artery catheters in high-risk surgical patients. N Engl J Med. 2003;348(1):5-14.

64. Schmid S, Kapfer B, Heim M, et al. Algorithm-guided goal-directed haemodynamic therapy does not improve renal function after major abdominal surgery compared to good standard clinical care: a prospective randomised trial. Crit Care. 2016;20:50.

65. Scheeren TWL, Wiesenack C, Gerlach H, et al. Goal-directed intraoperative fluid therapy guided by stroke volume and its variation in high-risk surgical patients: a prospective randomized multicentre study. J Clin Monit Comput. 2013;27(3):225-33.

66. Shoemaker WC, Appel PL, Kram HB, et al. Prospective trial of supranormal values of survivors as therapeutic goals in high-risk surgical patients. Chest. 1988;94:1176-86.

67. Smetkin AA, Kirov MY, Kuzkov W, et al. Single transpulmonary thermodilution and continuous monitoring of central venous oxygen saturation during off-pump coronary surgery. Acta Anaesthesiol Scand. 2009:53:505-14.

68. Srinivasa S, Taylor MH, Singh PP, et al. Randomized clinical trial of goaldirected fluid therapy within an enhanced recovery protocol for elective colectomy. Br J Surg. 2013;100(1):66-74.

69. Valentine RJ, Duke ML, Inman MH, et al. Effectiveness of pulmonary artery catheters in aortic surgery: a randomized trial. J Vasc Surg. 1998;27(2):203-11.

70. van Beest PA, Vos JJ, Poterman M, et al. Tissue oxygenation as a target for goal-directed therapy in high-risk surgery: a pilot study. BMC Anesthesiol. 2014;14:122.

71. Wakeling HG, MCFall MR, Jenkins CS, et al. Intraoperative oesophageal Doppler guided fluid management shortens postoperative hospital stay after major bowel surgery. Br J Anaesth. 2005;95:634-42.

72. Weineberg $L$, lanno $D$, Churilov $L$, et al. Restrictive intraoperative fluid optimisation algorithm improves outcomes in patients undergoing pancreaticoduodenectomy: a prospective multicentre randomized controlled trial. PLoS One. 2017;12(9):e0183313.

73. Wenkui $Y$, Ning $L$, Jianfeng $G$, et al. Restricted peri-operative fluid administration adjusted by serum lactate level improved outcome after major elective surgery for gastrointestinal malignancy. Surgery. 2010;147(4): 542-52.

74. Wilson J, Woods I, Fawcett J, et al. Reducing the risk of major elective surgery: randomised controlled trial of preoperative optimisation of oxygen delivery. BMJ. 1999;318:1099-103.

75. Wu J, Ma Y, Wang T, et al. Goal-directed fluid management based on the auto-calibrated arterial pressure-derived stroke volume variation in patients undergoing supratentorial neoplasms surgery. Int J Clin Exp Med. 2017; 10(2):3106-14

76. Xu H, Shu SH, Wang D, et al. Goal-directed fluid restriction using stroke volume variation and cardiac index during one-lung ventilation: a randomized controlled trial. J Thorac Dis. 2017;9(9):2992-3004.

77. Zakhaleva J, Tam J, Denoya PI, et al. The impact of intravenous fluid administration on complication rates in bowel surgery within an enhanced recovery protocol: a randomized controlled trial. Color Dis. 2013;15(7):892-9.

78. Zeng K, Li Y, Liang M, et al. The influence of goal-directed fluid therapy on the prognosis of elderly patients with hypertension and gastric cancer surgery. Drug Des Devel Ther. 2014;8:2113-9.

79. Zhang J, Chen CQ, Lei $X Z$, et al. Goal-directed fluid optimization based on stroke volume variation and cardiac index during one-lung ventilation in patients undergoing thoracoscopy lobectomy operations: a pilot study. Clinics. 2013;68(7):1065-70.

80. Zhang J, Qiao H, He Z, et al. Intraoperative fluid management in open gastrointestinal surgery: goal-directed versus restrictive. Clinics. 2012;67(10): 1149-55.

81. Ziegler DW, Wright JG, Coban PS, et al. A prospective randomized trial of preoperative optimization of cardiac function in patients undergoing elective peripheral vascular surgery. Surgery. 1997;122(3):584-92.

82. Wetterslev J, Thorlund C, Brok J, et al. Trial sequential analysis may establish when firm evidence is reached in cumulative meta-analysis. J Clin Epidemiol. 2008;61:64-75.

83. Park J. Postoperative kidney injury. Korean J Anesthesiol. 2017;70(3):258-66.

84. Singh P, Ricksten SE, Bragadottir G, et al. Renal oxygenation and haemodynamics in acute kidney injury and chronic kidney disease. Clin Exp Pharmacol Physiol. 2013;40(2):138-47.

85. Futier E, Lefrant JY, Guinot PG, et al. Effect of individualized vs standard blood pressure management strategies on postoperative organ dysfunction among high-risk patients undergoing major surgery: a randomized clinical trial. JAMA. 2017;318(14):1346-57.

86. Patel A, Prowle JR, Ackland GL, et al. Postoperative goal-directed therapy and development of acute kidney injury following major elective noncardiac surgery: post-hoc analysis of POM-O randomized controlled trial. Clin Kidney J. 2017;10(3):348-56.

87. Prowle J, Bellomo R. Fluid administration and the kidney. Curr Opin Crit Care. 2013;19:308-14.

88. Heßler M, Arnemann P, Ertmer C. To use or not to use hydroxyethyl starch in intraoperative care: are we ready to answer the'Gretchen question'? Curr Opin Anesthesiol. 2015;28(3):370-7.

89. Yates DR, Davies SJ, Milner HE, et al. Crystalloid or colloid for goal-directed fluid therapy in colorectal surgery. Br J Anaesth. 2014;112(2):281-9.

90. Joosten A, Delaporte A, Mortier J, et al. Long-term impact of crystalloid versus colloid solutions on renal function and disability-free survival after major abdominal surgery. Anesthesiology. 2019;130(2):227-36.

91. Ceba-Rartin R, Kashiouris M, Plataki M, et al. Risk factors for development of acute kidney injury in critically ill patients: a systematic review and metaanalysis of observational studies. Crit Care Res Pract. 2012;2012:691013.

92. Older $\mathrm{P}$, Smith R. Experience with the preoperative invasive measurement of hemodynamic, respiratory and renal function in 100 elderly patients scheduled for major abdominal surgery. Anesth Intens Care. 1988;16:389-95.

93. Mavrogenis A, Mitsiokapa EA, Romantini M, et al. Acute renal failure in orthopaedic surgery. J Long-Term Eff Med Implants. 2011;21(2):149-58.

94. Hassan BK, Sahlström A, Dessau RB. Risk factors for renal dysfunction after total hip joint replacement; a retrospective cohort study. J Orthop Surg Res. 2015;10:158.

95. Meersch M, Schmidt C, Hoffmeier A, et al. Prevention of cardiac surgeryassociated AKI by implementing the KDIGO guidelines in high risk patients identified by biomarkers: the PrevAKI randomized controlled trial. Intensive Care Med. 2017:43:1551-61.

96. Gocze I, Jauch D, Gotz M, et al. Biomarker-guided intervention to prevent acute kidney injury after major surgery the prospective randomized BigpAK Study. Ann Surg. 2018;267:1013-20.

\section{Publisher's Note}

Springer Nature remains neutral with regard to jurisdictional claims in published maps and institutional affiliations. 